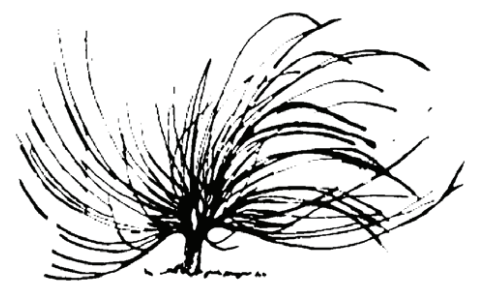

\title{
Impacto de la Formación Científica Secundaria en Dos Comunidades Indígenas Cabécar y Ngäbe
}

\author{
Giselle León León ${ }^{1}$ \\ Universidad Nacional \\ Heredia, Costa Rica \\ leongiselle@hotmail.com \\ Ericka Vargas Acuña ${ }^{3}$ \\ Universidad Nacional \\ Heredia, Costa Rica \\ ercka92@hotmail.es
}

Daniel Enrique Méndez Masis ${ }^{2}$

Universidad Nacional

Heredia, Costa Rica

dmendezmasis23@gmail.com

\begin{abstract}
Resumen
Este artículo expone los resultados de una investigación sobre el impacto a nivel de lengua, valores y tradiciones de la educación secundaria en biología para el nivel de quinto año en dos colegios de los territorios indígenas Cabécar y Ngäbe. La investigación se fundamentó en un enfoque mixto sobre todo a nivel del tipo de instrumentos. Para recopilar la información, se contó con el aporte de dos grupos de quinto nivel, uno de cada una de las comunidades en estudio y dos docentes de biología. Los resultados de los cuestionarios se muestran mediante un análisis descriptivo. Dentro de las principales conclusiones, se puede indicar que a pesar de que
\end{abstract}

Recibido: 18 de noviembre de 2013 - Aprobado: 18 de agosto de 2014

1 Académica de la División de Educología, Universidad Nacional de Costa Rica. Máster en Administración Educativa, Bachiller en la Enseñanza de las Ciencias y Licenciada en Ciencias de la Educación con Énfasis en Didáctica de Ciencias. Estudiante del Doctorado en Educación de la Universidad Autónoma de Durango, México.

2 Estudiante de enseñanza de las ciencias y biología, Universidad Nacional.

3 Estudiante de enseñanza de las ciencias, Universidad Nacional. 
la fundamentación de la educación propone desarrollar valores y tradiciones autónomos de la comunidad, son muy pocos los que realmente se vivencian. Por su parte, la forma en que el profesional de biología promueve la identidad es contextualizando en el proceso de mediación. De igual manera, el estudio arrojó la importancia de que las lecciones sean desarrolladas en su idioma nativo y por una persona Ngäbe o Cabécar.

Palabras clave: indígenas, Cabécar y Ngäbe, impacto, valores, tradiciones, lengua, asignatura biología

\begin{abstract}
This paper presents the results of a research about the impact of the language level, values, and traditions of secondary education in tenth grade Biology at two high schools located in Cabécar and Ngäbe indigenous territories. This research was based on a mixed approach, taking into account the different kinds of instruments used. Two groups of eleventh grade (students) and two biology teachers from two different high schools were surveyed in order to gather the information. The interviews' results are shown through a descriptive analysis. The main conclusions were the following: Although the educational system applied in these communities promotes the development of their autonomous values and traditions, only few of them are still present. Moreover, this research found out that biology professionals (teachers) promote the Indigenous identity by contextualizing the mediation process. Additionally, this research evinced the importance of speaking the indegenous students' native language in the classrooms and the importance of the lessons being taught by Cabécar or Ngäbe people.
\end{abstract}

Keywords: Indigenous people, Cabécar and Ngäbe, impact, values, traditions, language, biology 


\section{Introducción}

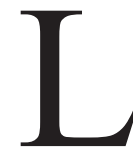

os pueblos indígenas han sido los herederos y los guardianes de un rico patrimonio cultural y natural, a pesar de haber sufrido, desde la época de la colonia y hasta hoy, el despojo de sus tierras, riquezas e incluso la negación de su identidad étnica, lingüística y cultural. No se puede negar que la globalización ha ayudado a mejorar la calidad de vida de los seres humanos, logrando traspasar fronteras religiosas, étnicas, lingüísticas, culturales, que antes parecían inquebrantables.

Del mismo modo, no se puede menospreciar la creciente tendencia de este sistema a homogenizar ideológica y culturalmente a los sujetos para predecir y controlar la acción humana. Lo indicado antes, como ya se mencionó tiene sus orígenes desde la colonia europea, debido a las creencias occidentales acerca de la inferioridad de estos pueblos indígenas. A lo largo de los años, se ha visto como las poblaciones indígenas han ido perdiendo su lengua sobre todo porque el flagelo de la globalización los ha ido marcando; en ese mismo marco, se encuentra el proceso educativo (Brenes, 2007).

En la actualidad, las comunidades indígenas costarricenses tienen acceso a la educación primaria y secundaria, mas las diferentes asignaturas son desarrolladas en español, dejando de lado su idioma tradicional. Razón por la cual muchos jóvenes y algunos adultos utilizan el castellano como lengua principal para comunicarse. Lo anterior aunado a la pérdida de sus tradiciones tales como su vestimenta, comidas, actividades, entre otros (Romero, 2007).

Ante esta realidad de pérdida de identidad cultural, se hace necesario una formación que permita desarrollar y promover su cultura. Por tal motivo, la investigación desarrollada persiguió analizar el impacto que tiene la educación costarricense brindada por el Ministerio de Educación Pública (MEP) en dos comunidades indígenas, Cabécar y Ngäbe, específicamente, en el desarrollo de las lecciones de biología, tomando en cuenta la lengua, valores y tradiciones de los dos grupos indígenas en estudio.

\section{Problema}

¿Cómo analizar el impacto a nivel de lengua, valores y tradiciones de la educación secundaria en biología para el nivel de quinto año en dos colegios de los territorios indígenas Cabécar y Ngäbe costarricenses? 


\section{Objetivo general}

Analizar el impacto a nivel de lengua, valores y tradiciones de la educación secundaria en la materia de biología para el nivel de quinto año en dos colegios de los territorios indígenas Cabécar y Ngäbe costarricenses.

\section{Objetivos específicos}

- Diagnosticar el impacto que la educación secundaria ha provocado sobre la población de dos colegios de los territorios indígenas Cabécar y Ngäbe en cuanto a la pérdida de valores y tradiciones.

- Describir la forma en que la persona profesional de biología promueve la identidad en el desarrollo de las lecciones.

- Identificar la importancia de que la formación secundaria sea desarrolla en el idioma nativo.

\section{Aspectos teóricos de referencia}

Uno de los elementos teóricos que guiaron el artículo fueron los valores, los cuales de acuerdo con Segura (2006), se definen como los principios que guían al ser humano en su convivencia en función de realizarlos como personas. De igual manera, se puede decir que los valores son la cualidad estructural que surge de la relación de un sujeto con un objeto, pero esta relación no se da en el vacío, sino en un contexto y en ocasiones, ese contexto ha sido ignorado o menospreciado para entender la cuestión axiológica.

Por otra parte, desde 1976-1977, se establecieron los límites territoriales de las tierras indígenas. De acuerdo con la información del censo 2011, en Costa Rica hay 24 territorios indígenas, en los cuales se encuentran distribuidos ocho pueblos indígenas. Uno de ellos es el Cabécar, específicamente, en el marco de la investigación, se tomaron en cuenta a los de Talamanca. Por su parte, los Ngäbes, los constituyen poblaciones migratorias de Panamá. Por tal motivo, se habla de los de Conte Burica.

Tomando como referencia el censo antes mencionado, la población Cabécar se caracteriza por habitar zonas montañosas, lejanas, contribuyendo a mantener rasgos culturales propios, tales como idioma, 
tradiciones espirituales y familiares. En cuanto a los Ngäbe, se indica que existen diferencias de idiomas con los de Panamá y que el $100 \%$ de la población habla ngäbere (Instituto Nacional de Estadística y Censo, 2011).

Para Giménez (2005), los términos cultura e identidad van de la mano, uno se construye a base del otro:

[N]uestra identidad sólo puede consistir en la apropiación distintiva de ciertos repertorios culturales que se encuentran en nuestro entorno social, en nuestro grupo o en nuestra sociedad (...) la primera función de la identidad es marcar fronteras entre un nosotros y los 'otros' (...). (p. 1)

Así la identidad, por su parte, es la exposición de lo que encierra una cultura y que la hace diferentes a otras.

En relación con las tradiciones, para Davini (citado por Calzón, 2006), es algo heredado, que va pasando de generación en generación, algo que se recibe al nacer. El ser humano no elige las tradiciones. En el marco del fenómeno de formación docente, las tradiciones son el conjunto de acciones y prácticas pedagógicas que se encuentran institucionalizadas.

Aunque parezca que las tradiciones cambian siempre, el núcleo de ellas se mantiene, es decir, la parte más importante de ellas se conserva. Lo que cambia es lo superficial. En cada época histórica siempre habrá una orientación que marcará el modo de acción que tendrán las prácticas pedagógicas.

En relación con el impacto de la globalización de los pueblos indígenas, Romero (2007) refiere que la globalización es entendida como la homogenización económica, cultural, social y política, promueve y genera valores y derechos universales y no responde a las demandas de los movimientos indígenas del mundo. Lo anterior puesto que al ser autónomos, es decir, los diferentes grupos indígenas tienen sus propias dogmas, ideologías y tipos de vida, por ende, cada vez son más excluidos, ya que el marco de globalización justamente persigue uniformar a la luz de lo que dictan las potencias, razón por la cual las ideas propias, formas de vida y creencias no tienen cabida. La idea anterior es afirmada por Bello, 2006 al indicar que

[1]a dinámica económica de la globalización tiende a favorecer a los actores que poseen el poder político y económico para 
proponer los términos del intercambio neoliberal donde amplios sectores son marginados o incluidos de manera subordinada. La conciencia étnica, sin embargo ha buscado los modos de representar su descontento con las condiciones actuales de la globalización económica y sus implicancias políticas en la medida que ha percibido dichos procesos como contrarios a sus intereses y demandas. (p. 2).

Tal como se indicó antes, desde la perceptiva económica, los pueblos indígenas han dado su lucha, pero hasta el momento son escasos los avances, razón por la cual se puede decir que el marco de la globalización ha aumentado las desigualdades y los desequilibrios sociales, políticos y económicos de los pueblos indígenas.

Haciendo alusión al proceso educativo, Peña (1997) indica que el perfil de un docente intercultural debe contemplar tanto aptitudes como "actitudes habituales-autenticas virtudes". Él menciona principalmente tres: la primera de ellas hace referencia al conocimiento que debe poseer la persona profesional de la educación, de la cultura en la que se encuentra, sus problemas, logros y objetivos, para así poder ayudar en conjunto, pero ello principalmente transmitiéndolo a sus educandos.

Por otro lado, no basta con saber, sino ser partícipe del medio, sentir no sólo la necesidad de transmitir sino vivirlo, lo que involucra que debe ser abierto y tolerante a otras culturas. Y como tercera actitud, y quizá la más difícil, estar abierto al cambio, a las innovaciones, pero desde su papel como docente, velando porque los dos aspectos no sean sinónimo de pérdida de valores, de la identidad, sino que vayan de la mano. Y como aptitudes menciona la competitividad, mediante una formación constante, capacidad para realizar investigación-acción del contexto educativo y, finalmente, dominar tanto la lengua del país así como la lengua materna de sus educandos.

De acuerdo con la Asamblea Legislativa de la República de Costa Rica, en el Convenio de Pueblos Indígenas y Tribales en Países Independientes, en el artículo 27, inciso 1:

Los programas y los servicios de educación destinados a los pueblos interesados deberán desarrollarse y aplicarse en cooperación con éstos a fin de responder a sus necesidades particulares, y deberán abarcar su historia, sus conocimientos y técnicas, sus 
sistemas de valores y todas sus demás aspiraciones sociales, económicas y culturales. (s. p.)

Por su parte, el Artículo 28 incisos 1 y 3 indican que:

[D]eberá enseñarse a los niños de los pueblos interesados a leer y a escribir en su propia lengua indígena o en la lengua que más comúnmente se hable en el grupo a que pertenezcan. Cuando ello no sea viable, las autoridades competentes deberán celebrar consultas con esos pueblos con miras a la adopción de medidas que permitan alcanzar este objetivo. Deberán adoptarse disposiciones para preservar las lenguas indígenas de los pueblos interesados y promover el desarrollo y la práctica de las mismas. (s. p.)

Para diseñar materiales didácticos a base de los recursos naturales con los que cuenta una zona indígena, es necesario contemplar qué tanto conocen los educandos y qué papel juegan estos en sus comunidades. Estos dos puntos fueron los empleados por Cebrián y Noguera en una investigación publicada en el 2010 y llevada a cabo en Venezuela. Ellos no solo sondearon a la población sino que la hicieron patícipe de la creación del material didáctico. Para ellos, sería primordial que cada docente construyera su material, además que debería encontrarse en la lengua nativa de cada región.

Las personas están inmersas en una realidad social, su desarrollo personal no puede disociarse del intercambio con ella, su personalidad se va forjando en su participación, en las creencias, actitudes, comportamientos de los grupos a los que pertenece. Esa realidad colectiva consiste en un modo de sentir, comprender y actuar en el mundo y en formas de vida compartidas, que se expresan en instituciones, comportamientos regulados; en suma en lo que se entiende por una cultura. El problema de identidad de los pueblos remite a su cultura (Villoro, s. f.).

\section{Metodología}

\section{Paradigma}

La investigación se llevó a cabo bajo un paradigma naturalista, también llamado naturalista-humanista o interpretativo, el cual "se centra en el estudio de los significados de las acciones humanas y de la vida social" 
(Barrantes, 2010, p.60). Al tomar en cuenta la investigación, el impacto del proceso educativo desarrollado en secundaria en dos poblaciones indígenas, se determinó la pertenencia de dicho trabajo en este paradigma.

\section{Enfoque de estudio}

La presente investigación se ubica dentro del modelo de enfoque dominante, el cual sustentan Hernández, Fernández y Baptista (2006): "el estudio se desarrolla bajo la perspectiva de alguno de los dos enfoques, la cual prevalece, y la investigación mantiene un componente del otro enfoque" (p. 773). Para esta investigación, se utilizó el enfoque dominante cualitativo, con algunos elementos de corte cuantitativo, específicamente en el diseño de instrumentos.

\section{Tipo de estudio}

El tipo de estudio, en el cual se enfocó está investigación fue etnográfica, específicamente como la llama Paz (2003) etnográfica educativa. Ésta hace referencia a la interacción propiamente en el marco educativo entre grupos sociales y culturales. Busca explicar fenómenos desde la opinión de los sujetos. Por ello, la presente investigación corresponde a este tipo de estudio, ya que buscó encontrar el papel que ha jugado la educación en el fortalecimiento de valores y tradiciones, así como de la identidad, tomando en cuenta la opinión directa de las personas y situándose el investigador en el contexto.

\section{Participantes}

El estudio se llevó a cabo en dos territorios indígenas de Costa Rica, Talamanca Cabécar y los Ngäbe en Conte Burica. En cada uno de ellos, se seleccionó a las personas estudiantes que cursaban el quinto año en el momento del estudio ${ }^{4}$, así como a su respectivo docente de biología. Para los de Talamanca Cabécar, la muestra fue de siete educandos y una persona docente, mientras que en Conte Burica, la población fue de 15 educandos y una persona profesional de las ciencias naturales.

\section{Instrumentos y técnicas utilizadas}

Como primer paso, se realizó una observación en ambos lugares, tanto del contexto social como del educativo, a partir de la cual

4 II ciclo del año 2013. 
surgió el tema de investigación. Para llevar a cabo la investigación, se empleó como principal instrumento el cuestionario. Uno dirigido a los estudiantes y las estudiantes, constituido por 16 preguntas y otro fue diseñado para la persona profesional de biología, el cual constó de 11 ítems. Ambos cuestionarios contaban con preguntas abiertas y cerradas. Con ellos se indagó acerca de las categorías del estudio: impacto de la educación secundaria (valores-tradiciones), fomento de la identidad cultural por parte del docente e importancia de que la formación secundaria sea desarrolla en el idioma nativo.

\section{Consideraciones éticas}

Para recopilar la información, se solicitó la ayuda tanto a educandos como docentes. De igual manera, se les explicó el objetivo del trabajo.

\section{Análisis}

En este apartado, se realiza una descripción detallada de las principales respuestas que se obtuvieron a partir de los instrumentos aplicados a las personas informantes. En primera instancia, se presentan los resultados obtenidos en cuanto a valores y tradiciones. En un segundo momento, se aborda el implemento de la identidad en las lecciones de biología, por último, la importancia de que la formación secundaria sea desarrolla en el idioma nativo.

De acuerdo con lo referido por docentes y educandos del territorio de Talamanca, en las clases de ciencias se promovían valores tales como cortesía, responsabilidad, crítica constructiva, solidaridad, amistad y sencillez. Por el contrario, un estudiante de los encuestados indicó que el único valor promovido es la responsabilidad y expresó que los otros "podrían ser", ya que no son tan evidentes.

Con respecto a la promoción de valores en la comunidad de Comte en las clases de ciencias por parte del docente, los educandos consideran que de los cinco valores antes mencionados, el que no se promueve es la responsabilidad por parte del docente. Tomando en cuenta lo anterior, se puede decir que sí se está propiciando lo que se indica a nivel teórico, ya que la proposición de valores permite un intercambio en un espacio social (Segura, 2006), espacio desarrollado en las lecciones de biología y ratificado por las personas encuestadas.

En cuanto a las interrogantes relacionadas con los días festivos, vestimenta particular y religión propios de cada territorio, los y las 
estudiantes de la población Ngäbe consideraron, en su mayoría, que en su cultura, sí hace uso de vestimenta propia, la cual los identifica como un pueblo indígena. Asimismo, se destaca lo manifestado por todas las mujeres encuestadas de la comunidad de Comte, quienes indicaron poseer y utilizar vestimenta alusiva a su grupo indígena. Lo descrito anteriormente se pudo constatar mediante las observaciones realizadas por el colectivo investigador. Por su parte, la población Cabécar refirió no poseer ninguna vestimenta que los identifique como parte de una población indígena.

La muestra de educandos encuestados coincidió en que poseen un día festivo propio de su cultura, mencionaron el 12 de octubre, aunque la pregunta iba inclinada a un día festivo que los identificara más como población. Con respecto a la religión autónoma, tanto los Ngäbe y Cabécar indicaron que no se práctica ninguna religión propia de su cultura. Mas los Ngäbe, dicen practicar una religión oriental, la cual se llama la Fé bahais.

Con respecto al fortalecimiento de la identidad en el desarrollo de las lecciones de biología, tanto la persona profesional de biología, como los educandos de la comunidad de Talamanca refirieron que la formación en el área de ciencias (biología) fortalece su identidad mediante el uso de la flora y fauna de la zona.

Por su parte, los educandos agregaron que la persona docente hace uso de su lengua nativa en algunos ejemplos. Sin embargo, un encuestado expresó que los ejemplos utilizados por su profesor(a) son muy generales, de igual manera, agregó que en ocasiones utiliza términos cuyo significado desconoce.

A propósito de lo recién indicado, la muestra de educandos de la comunidad Comte en su mayoría refirieron que sí se promueve el fortalecimiento de la identidad, ya que su profesor(a), al explicar algún concepto, hace referencia a ejemplos sobre el entorno que los rodea, también algunas veces expone ejemplos propios de la cultura Ngäbe. Sin embargo, resaltaron la falta de espacios en las lecciones para fortalecer más la cultura e identidad por parte del docente. Lo anterior lo afirman Cebrián y Noguera (2010), al indicar que la solidificación de la identidad en los individuos va estrechamente relacionada con el contexto existente a su alrededor y este es uno de los objetivos buscados por el sistema educativo. 
En lo que concierne al uso de tecnologías, específicamente, el internet en las clases, todos(as) los encuestados y las encuestadas consideraron este como un medio que se puede emplear en las clases de biología. En su opinión, la implementación de este recurso ayudaría en la búsqueda de información en general y para ampliar los trabajos extra clase, ya que no cuentan con biblioteca para consultar libros para sus tareas o trabajos de investigación. Según lo mencionado por Cebrián y Noguera (2010), el internet suele ser, más que un medio fortalecedor de la identidad, una razón directa de la pérdida de ella. A pesar de las justificantes que indicaron los educandos, ciertamente, el internet es una herramienta generada en el entorno de la globalización, lo señalado antes es confirmado por uno de los docentes del estudio: "es una herramienta útil, pero ésta ha favorecido la perdida de la cultura".

\section{Al respecto García (2006) expone:}

Existen ambientes de aprendizajes que tienen las condiciones pedagógicas y contextuales donde el conocimiento es el factor principal para formar seres reflexivos y autodidactas, pero para lograr este objetivo es necesario que sean los mismo estudiantes quienes estén convencidos que la tecnología es una herramienta para lograr el gran reto de la educación, es necesario que los estudiantes manifiesten una actitud crítica y práctica en el uso de estos nuevos medios porque es precisamente la computadora-Internet un sistema virtual, la herramienta de trabajo para la apropiación del conocimiento (...). (p. 6)

En cuanto a la interrogante referida a la importancia de que el docente sea Ngäbe o Cabécar, se identificaron opiniones encontradas. Por ejemplo, tres de las siete personas estudiantes (Cabécar) opinaron que no es importante, ya que lo realmente importante es que el o la docente tenga el conocimiento para impartir las clases.

Por su parte, los educandos de la comunidad de Comte indicaron mayoritariamente la importancia de que el profesional sea Ngäbe. Algunas de sus justificantes fueron: mejor desarrollo de las clases, espacio para practicar el idioma, de igual manera, para los que aún no conocen el lenguaje puedan aprenderlo de manera que no se pierda, pues son pocas personas las que lo hablan; cabe añadir que algunos educandos 
coincidieron con lo referido por el estudiantado de la muestra Cabécar, al expresar que lo más significativo es que el colectivo docente esté bien preparado y conozca la materia. Para Rebolledo (s.f.):

...la identidad del profesor indígena bilingüe es, como se ha visto, un componente básico para el desarrollo de la educación en las regiones interétnicas. La atención de los grupos escolares multigrado y la diversidad lingüística exige un esfuerzo por parte del profesor. (p. 25)

Por su parte, el colectivo docente de la investigación mencionó que sí es importante que la persona que se desempeñe como guía del proceso educativo sea parte de la comunidad indígena, lo anterior, puesto que puede enriquecer las clases y entender más la realidad de vida, por ende, el proceso de formación de los educandos.

Por su parte, la persona docente de Talamanca Cabécar dio como justificante de la importancia de que el docente sea parte de territorio indígena para evitar la pérdida de la identidad. Esto último es considerado por Ipiña (1997), como una de las condiciones que debe cumplir todo educador intercultural, y la clasifica como una primera actitud denominada "compromiso con las causas del pueblo" (p. 103).

En relación con que se imparta una asignatura correspondiente al idioma nativo, el estudiantado Cabécar de la muestra refirió opiniones en su mayoría positivas, aduciendo la importancia de que no se pierda su lengua, ya que algunos jóvenes de la comunidad no la utilizan.

En contraste, las personas con una posición negativa opinaron que no es relevante, pues el idioma ya casi no se utiliza, además de tener limitantes de palabras, ya que algunas utilizadas en el español no existen en Cabécar, aunado a que no hay individuos en capacidad de transmitir sus conocimientos de lengua. En ese sentido, Ipiña (1997) menciona que una solución a que casi no haya personas preparadas, sería la formación de jóvenes propios de cada zona, por lo tanto, conocedores de su cultura, pues no es fácil formar buenos docentes en educación intercultural bilingüe.

En relación con esa misma interrogante, el colectivo estudiantil de la zona sur Ngäbe, de igual manera, expresó la trascendencia de que se imparta una asignatura correspondiente a su idioma, puesto que les permitiría rescatar su cultura, principalmente para las futuras 
generaciones. Según expone López (s.f.), “el aprendizaje, la enseñanza y el uso de las lenguas indígenas en la escuela enriquecen la educación de los educandos indígenas, además contribuyen a un mejor rendimiento escolar de los mismos" (p. 15), por lo que el empleo de la lengua nativa no sólo ayudaría a que ella no se pierda, además favorecería el desempeño escolar.

Por otro lado, todos(as) los educandos y las encuestadas consideraron que sí es importante obtener el título en Bachillerato en Educación Media, pues les puede permitir prosperar y con ello tener una mejor calidad de vida, al poder optar por un trabajo. Asimismo, les permitiría cursar una carrera universitaria, razón que es igualmente compartida por las personas profesionales de ciencias. Para Treviño (2006), más que la lucha o deseo del estudiantado por un título, analiza los obstáculos para obtenerlo y la injusticia que hay detrás de pruebas estandarizadas y lo que ellas significan, pues en algunos casos están creadas como filtros, que impiden el crecimiento de los educandos al ver imposibilitada la opción de optar por estudios superiores, pues simplemente no aprueban el examen con una nota que les permita ingresar a la universidad.

\section{Conclusiones}

El proceso educativo desde su fundamento promueve el fortalecimiento de valores y tradiciones. Sin embargo, se pudo identificar como en las dos comunidades Cabécar y Ngäbe se promueven principalmente valores como cortesía, responsabilidad, crítica constructiva, solidaridad, amistad y sencillez. En cuanto a tradiciones, se determinó que son muy pocas las tradiciones propias que se promueven en el marco de las lecciones de biología, se resalta el uso de la vestimenta por parte de las mujeres Ngäbe, esa misma población es la que más utiliza su idioma para comunicarse entre los compañeros(as) en el desarrollo de las lecciones.

En cuanto a la promoción de la identidad por parte del docente de biología, se determinó en ambos casos que la persona docente al menos trata de utilizar la naturaleza de la zona como recurso didáctico, por ejemplo, nombres de animales propios de la zona, así como implementar el respeto y cuidado de los recursos naturales. Sin embargo, los educandos de ambas comunidades en su mayoría consideran que sería útil y agradable el uso del internet como parte del proceso de mediación 
pedagógica, elemento que, si no es usado de manera adecuada, puede aunar a la pérdida de identidad.

Con respecto a la promoción de su idioma, se reflejó en las dos comunidades el interés en que las lecciones sean impartidas en su lenguaje nativo por una persona Cabécar o Ngäbe, puesto que esto permitiría desarrollar su identidad, tanto a nivel de tradiciones, valores, como del idioma, unido a que sea un docente con un buen dominio de la materia.

\section{Referencias bibliográficas}

Asamblea Legislativa de la República de Costa Rica. (s.f.). Ley de aprobación del Convenio $N^{\circ} 169$ sobre Pueblos Indígenas y Tribales en Países Independientes.Recuperado de http://ministeriopublico.poder-judicial.go.cr/coop-intern/ inst-inter/18/01.pdf

Barrantes, R. (2010). Investigación: un camino al conocimiento un enfoque cualitativo y cuantitativo. Costa Rica, San José: EUNED.

Bello, A. (2006).Pueblos indígenas, globalización y territorio. Recuperado de http:// www.observatorio.cl/sites/default/files/biblioteca/doc_trabajo_numero4-pueblos-indigenas-glob-y-terri.pdf

Brenes, C. (2007). Tecnologías de información y comunicación: El caso de las comunidades indígenas Cabécares de Chirripó de Costa Rica. Revista Educare,1(17), 177-192.

Calzoni, L. (2006). El concepto de "Tradición” en la formación docente la tradición "Normalizadora-Disciplinadora". Recuperado de http://www.cerpcentro.org/ IMG/pdf/Concepto_de_tradicion_-_Davini.pdf

Cebrián, M. y Noguera, J. (2010). Conocimiento indígena sobre el medio ambiente y diseño de materiales educativos. Comunicar: Revista Científica Iberoamericana de Comunicación y Educación. 34(17), 115-124. Recuperado de http://dialnet. unirioja.es/servlet/articulo?codigo $=3166269$

García, M. (2006). La tecnología en las comunidades indígenas. Recuperado de http:// www.oei.es/memoriasctsi/mesa9/m09p04.pdf

Giménez, G. (2005). La cultura como identidad y la identidad como cultura. III Encuentro internacional de Promotores y Gestos Culturales, Guadalajara, México.

Hernández, R., Fernández, C. y Baptista, P. (2006). Metodología de la investigación (4 Ed.). México, México DF: McGrawHill.

Instituto Nacional de Estadística y Censo. (abril, 2013). X Censo Nacional de Población y VI de Vivienda 2011.Territorios Indígenas Principales indicadores demográficos y socioeconómicos. Recuperado de ttp://accionsocial.ucr. ac.cr/c/document_library/get_file?uuid=f0c2fc79-443f-4cb9-ba62-423891c54 $7 \mathrm{f} 2$ \&groupId $=23760$

Ipiña. E. (1997). Condiciones y perfil del docente de educación intercultural bilingüe. Revista Iberoamericana de Educación, 13, 99-109. Recuperado de http://www. aulaintercultural.org/IMG/pdf/rie13a04.pdf 
López, L. (s.f.). La cuestión de la interculturalidad y la educación latinoamericana. Recuperado de http://www.schwartzman.org.br/simon/delphi/pdf/lopes.pdf

Paz, M., (2003). Investigación cualitativa en educación. Madrid, España: McGraw-Hi11/Interamericana de España, S. A. U.

Peña, E. (1997). Condiciones y perfil del docente de educación intercultural bilingüe. Revista Iberoamericana de Educación, 13, 99-102.

Rebolledo, N. (s.f.). La formación de profesores indígenas bilingües en México. Recuperado de http://bibliotecadigital.conevyt.org.mx/servicios/hemeroteca/075/075004.pdf

Romero, G. (2007). La globalización: Una plataforma de exclusión de los pueblos indígenas. La paz-Bolivia: Fondo Indígena.

Segura, M. (2006). Los valores en el planeamiento didáctico eje transversal del currículo costarricense. San José, Costa Rica: MEP. Recuperado de http://www.educadem. oas.org/documentos/boletin3/valoresyplaneamiento\%20costa\%20rica.pdf

Treviño, E. (2006). Evaluación del aprendizaje de los estudiantes indígenas en América Latina. Revista Mexicana de Investigación Educativa, 28(11), 225-268.

Villaro, L. (s.f.). Identidad de los pueblos. Recuperado de http://interculturalidadffyl. files.wordpress.com/2011/09/villoro_identidad.pdf 